\title{
Kasuistiken
}

https://doi.org/10.1007/s00105-021-04812-6

Angenommen: 23. März 2021

Online publiziert:21. April 2021

(c) Der/die Autor(en) 2021
Hautarzt 2022 $\cdot 73: 152-155$

\author{
Susanne Hanner ${ }^{1} \cdot$ Hannah Stroh ${ }^{2}$ A Alexander Enk' · Jochen Hoffmann' \\ ' Hautklinik, Universität Heidelberg, Heidelberg, Deutschland \\ ${ }^{2}$ Haut- und Laserzentrum Heidelberg, Dres. Durani, Heidelberg, Deutschland
}

\section{Kutane Pseudolymphome nach Hirudotherapie}

\section{Fallbericht und Literaturübersicht}

\section{Anamnese}

Eine 76-jährige Patientin stellte sich mit seit über 1 Jahr bestehenden, juckenden, lividen bis erythematösen Plaques an der rechten Ferse sowie im Bereich des unteren Rückens in unserer allgemeinen Ambulanz vor. Auf intensive Nachfrage berichtete die Patientin, dass sie sich aufgrund von Gelenkschmerzen im Lendenwirbelsäulenbereich und eines Fersensporns einige Wochen vor Auftreten derEffloreszenzen 2 Sitzungen einer Blutegeltherapie durch eine Heilpraktikerin unterzogen hatte. Es wurden keine relevanten Nebenerkrankungen angegeben. Die Dauermedikation bestand aus Metoprolol, Ramipril sowie L-Thyroxin.

\section{Diagnostik}

Hautbefund Bei der Ganzkörperinspektion zeigten sich an oben genannten Ansatzstellen der Blutegel insgesamt 7 livide bis erythematöse, infiltrierte Plaques
(- Abb. 1a). Das übrige Integument und die Schleimhäute kamen unauffällig zur Darstellung. Zurweiteren diagnostischen Einordnung erfolgte die Durchführung einer Probebiopsie.

Histopathologischer Befund Es zeigten sich knotige, lymphozytär dominierte Entzündungsinfiltrate mit vereinzelten eosinophilen Granulozyten und Plasmazellen um eine zentral gelegene, zur Epidermis senkrecht stehende Narbenzone. Zur Tiefe hin waren regelrecht ausgebildete, reaktive Follikelzentren mit Kerntrümmermakrophagen, zahlreichen Mitosen und erhaltener Mantelzone erkennbar (• Abb. 1b, c).

Immunhistochemischer Befund Immunhistochemische Färbungen zeigten ein gemischtzelliges Infiltrat mit Nachweis von zahlreichen kleinen B- und T-Lymphozyten (Positivität für CD4und CD20).
Laborbefund Die Lues- und Borrelienserologie waren negativ.

\section{Diagnose}

In Zusammenschau der Anamnese, des klinischen Befundes, der Histopathologie sowie der Immunhistochemie stellten wir die Diagnose kutaner Pseudolymphome (PSL) nach medizinischer Blutegeltherapie.

\section{Therapie und Verlauf}

Es wurde eine topische Behandlung mit Mometason eingeleitet. Bei der letzten Wiedervorstellung zur Verlaufskontrolle nach 24 Monaten zeigten sich nur noch dezent sichtbare livide Verfärbungen im Bereich der ehemaligen Knoten an der Lendenwirbelsäule.
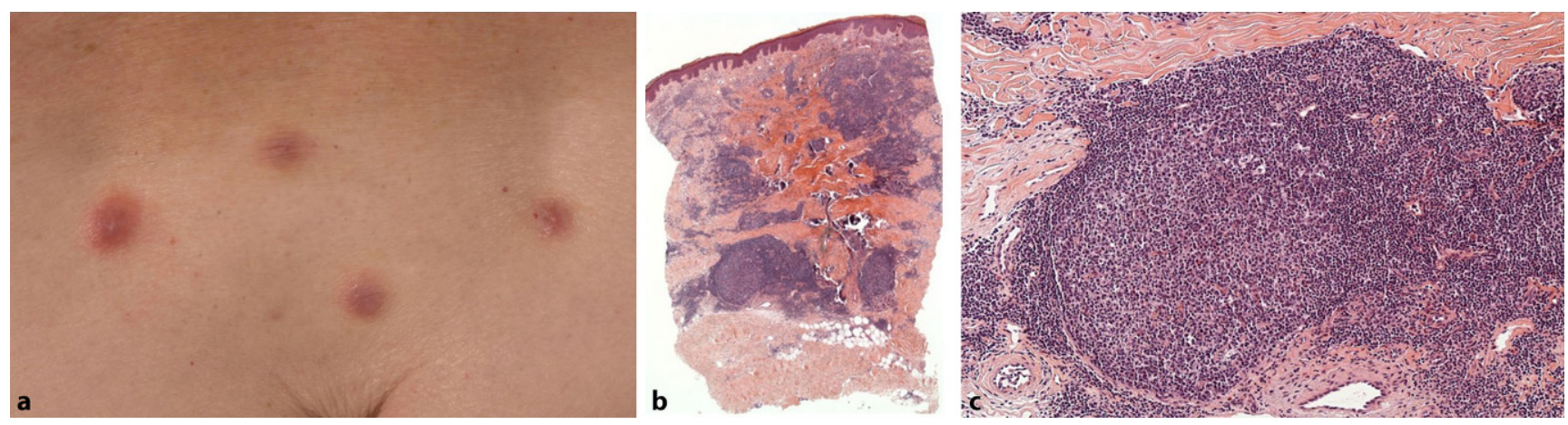

Abb. 1 ॥ Pseudolymphome nach Hirudotherapie. a Livid bis erythematöse Knoten und Plaques am unteren Rücken nach Hirudotherapie. b Histopathologie (Hämatoxylin-Eosin, Originalvergr. 2,5:1): dermale, noduläre, lymphozytär dominierte Infiltrate mit Ausbildung von reaktiven Follikelzentren um eine zentrale Narbe. c Histopathologie (Hämatoxylin-Eosin, Originalvergr. 10:1): reaktives Follikelzentrum mit Kerntrümmermakrophagen und erhaltener Mantelzone 


\section{Diskussion}

Unter dem Begriff Pseudolymphom (PSL) oder kutane lymphoide Hyperplasie versteht man eine benigne, reaktive Lymphoproliferation der Haut, die klinisch und/oder histologisch ein malignes Lymphom simulieren kann [1, 2]. Es handelt sich um eine heterogene Gruppe von Entitäten, die sich klinisch, histologisch, immunhistochemisch sowie ätiologisch unterscheiden [1]. In den letzten Jahren wurden in der Literatur zahlreiche Klassifikationen für Pseudolymphome publiziert [1]. Diese beinhalten Einteilungen der PSL nach dem Immunphänotyp (T-Zell-, B-Zelloder gemischtzellig), den histopathologischen Merkmalen, der Ätiologie sowie nach unterschiedlichen klinischen Merkmalen [2]. Eine international etablierte, konsensusbasierte Klassifikation analog der WHO(World Health Organization)EORTC(European Organization for Research and Treatment of Cancer)-Klassifikation für kutane Lymphome liegt bis zum jetzigen Zeitpunkt nicht vor [1]. Man unterscheidet die primären, idiopathischen PSL ohne erkennbare Ursache von den sekundären PSL mit bekanntem Stimulus [3, 4]. Triggerfaktoren umfassen u.a. verschiedene Medikamente und Infektionen, aber auch Traumata wie Tattoos, Insektenstiche, Akupunktur und Impfungen [2, 4-6]. Ein typisches histologisches Merkmal der kutanen PSL ist das Vorhandensein eines polyklonalen, lymphozytären Infiltrats in der oberen Dermis [4]. Charakteristisch für die PSL ist ein gutartiger Verlauf. Aufgrund der selten beschriebenen malignen Transformation sowie der mitunter erheblichen differenzialdiagnostischen Schwierigkeiten sollten regelmäßige Nachsorgeuntersuchungen erfolgen [1, 5].

In dem vorliegenden Fall kam es zu einem Auftreten von Pseudolymphomen nach einer Behandlung mit medizinischen Blutegeln (Hirudotherapie). Bisher wurden nach bestem Wissen und Gewissen insgesamt nur 9 Fälle von kutanen PSL nach Hirudotherapie in der Literatur beschrieben [3-11], die in - Tab. 1 zusammengefasst sind. Histologisch und immunhistochemisch zeigte sich ein breites Bild ohne Dominanz eines Musters. Therapeutisch wurde zumeist entweder eine topische oder intraläsionale Steroidbehandlung durchgeführt. Hierunter kam es in den berichteten Fällen zu einer Befundbesserung.

Im letzten Jahrzehnt gewann die Blutegeltherapie mit neuen Einsatzbereichen an zunehmender Bedeutung [8]. Die Hauptindikationen der medizinischen Blutegeltherapie stellen unterschiedliche Gelenkerkrankungen wie Osteoarthritis und Epikondylitis, Venenerkrankungen sowie die Lappenplastik der plastischen Chirurgie dar [12]. Die Blutegel umfassen mehr als 600 verschiedene Arten, der bekannteste und in der Medizin vorrangig eingesetzte Vertreter ist der Hirudo medicinalis $[6,12]$. Bis dato konnten mehr als 20 bioaktive Substanzen im Speichelsekret der Blutegel mit u.a. analgetischen, antiinflammatorischen, plättchenhemmenden, gerinnungshemmenden und Thrombin-regulatorischen Funktionen sowie mit antimikrobiellen und extrazellulären Matrix-abbauenden Wirkungen identifiziert werden [12]. Mögliche Komplikationen sind Infektionen, Blutungen, Anämie, allergische Reaktionen sowie Narbenbildung [3]. Zudem können - wie im dargelegten Fall - kutane PSL als seltene, aber beachtenswerte Nebenwirkungen der Blutegeltherapie auftreten. Die Pathogenese der kutanen PSL nach Hirudotherapie ist bis heute nicht gänzlich geklärt. Die pseudolymphomatösen Reaktionen werden vermutlich durch den Biss der scharfen Zähne, durch Substanzen der Blutegel oder durch infektiöse Erreger, die während der Behandlung übertragen werden, bedingt $[6,8]$. Als möglicher Pathomechanismus wird eine verzögerte Hypersensitivitätsreaktion auf Bestandteile der Insekten diskutiert [3]. In der Literatur wurden selten sowohl irritative als auch allergische Kontaktdermatitiden nach medizinischer Blutegeltherapie beschrieben [13]. Hirudin kommt als ein mögliches, auslösendes Agens in Betracht [13]. Die definitive Identifikation des auslösenden Agens ist bei mehr als 100 nachgewiesenen Proteinen mit einem molekularen Gewicht zwischen 10 und $97 \mathrm{kD}$ im Speichelsekret der Blutegel allerdings schwierig [13].
Hautarzt 2022 $\cdot 73: 152-155$

https://doi.org/10.1007/s00105-021-04812-6

(c) Der/die Autor(en) 2021

S. Hanner · H. Stroh · A. Enk · J. Hoffmann

Kutane Pseudolymphome

nach Hirudotherapie.

Fallbericht und Literaturübersicht

\section{Zusammenfassung}

Unter dem Begriff Pseudolymphom (PSL) versteht man eine benigne, reaktive Lymphoproliferation der Haut, die klinisch und/oder histologisch ein malignes Lymphom simulieren kann. Die genaue Ätiopathogenese ist bis heute nicht gänzlich geklärt. Man unterscheidet die primären, idiopathischen PSL ohne erkennbare Ursache von den sekundären PSL mit bekanntem Stimulus. Wir berichten über das Auftreten von Pseudolymphomen nach einer Behandlung mit medizinischen Blutegeln (Hirudotherapie). Bisher wurden nach bestem Wissen und Gewissen insgesamt nur 9 Fälle von kutanen PSL nach Hirudotherapie in der Literatur beschrieben.

Schlüsselwörter

Blutegeltherapie · Lymphoproliferation

- Histologie · Immunhistochemie .

Molekularpathologie

Cutaneous pseudolymphoma after hirud otherapy. Case report and review

\section{Abstract}

The term cutaneous pseudolymphoma (C-PSL) is defined in the literature as a benign, reactive lymphoproliferation that clinically and/or histopathologically imitates cutaneous lymphoma. The exact etiopathogenesis has not been fully elucidated to date. A distinction is made between primary, idiopathic PSL without an identifiable cause and secondary PSL with a known stimulus. We report the occurrence of pseudolymphoma after treatment with medicinal leeches (hirudotherapy). To the best of our knowledge, a total of only nine cases of cutaneous PSL after hirudotherapy have been reported in the literature to date.

Keywords

Medicinal leech therapy $\cdot$ Lymphoproliferation · Histology · Immunochemistry . Molecular pathology 


\section{Kasuistiken}

\begin{tabular}{|c|c|c|c|c|c|c|}
\hline $\begin{array}{l}\text { Autor (Jahr, } \\
\text { Referenz) }\end{array}$ & $\begin{array}{l}\text { Alter/Ge- } \\
\text { schlecht }\end{array}$ & Lokalisation & $\begin{array}{l}\text { Indikation der Hirudo- } \\
\text { therapie }\end{array}$ & Immunhistochemie & Therapie & Follow-up \\
\hline $\begin{array}{l}\text { Hanner et al. } \\
\text { (aktueller Fall) }\end{array}$ & $76 / w$ & $\begin{array}{l}\text { Unterer Rücken } \\
\text { (lumbal), Ferse }\end{array}$ & $\begin{array}{l}\text { Gelenkschmerzen, Fersen- } \\
\text { sporn }\end{array}$ & $\begin{array}{l}\text { Gemischt T- und B-lympho- } \\
\text { zytäres Infiltrat }\end{array}$ & Topische ST & $\begin{array}{l}\text { Langsam rückläufige } \\
\text { Läsion }\end{array}$ \\
\hline \multirow{3}{*}{$\begin{array}{l}\text { Sepaskhah et al. } \\
(2020),[11]\end{array}$} & \multirow[t]{3}{*}{$44 / w$} & \multirow[t]{3}{*}{ Schienbein } & \multirow[t]{3}{*}{ Erythema nodosum } & \multirow[t]{3}{*}{ Nicht durchgeführt } & Systemische ST & \multirow[t]{3}{*}{ Rückläufige Läsionen } \\
\hline & & & & & Topische ST & \\
\hline & & & & & Intraläsionale ST & \\
\hline \multirow{2}{*}{$\begin{array}{l}\text { Sadati et al. } \\
\text { (2019), [9] }\end{array}$} & \multirow[t]{2}{*}{$45 / w$} & \multirow{2}{*}{$\begin{array}{l}\text { Untere Extremi- } \\
\text { täten }\end{array}$} & \multirow[t]{2}{*}{ Varikose und Schmerzen } & \multirow[t]{2}{*}{ Nicht durchgeführt } & Topische ST & \multirow{2}{*}{$\begin{array}{l}\text { Komplett rückläufige } \\
\text { Läsionen }\end{array}$} \\
\hline & & & & & Kryotherapie & \\
\hline \multirow[t]{2}{*}{$\begin{array}{l}\text { Temiz et al. } \\
\text { (2019), [5] }\end{array}$} & \multirow[t]{2}{*}{$54 / \mathrm{m}$} & \multirow[t]{2}{*}{ Hals } & \multirow[t]{2}{*}{ Unbekannt } & \multirow[t]{2}{*}{$\begin{array}{l}\text { T-lymphozytär dominiertes } \\
\text { Infiltrat }\end{array}$} & \multirow[t]{2}{*}{ Intraläsionale ST } & $\begin{array}{l}\text { Nach } 1 \text { Monat: rückläu- } \\
\text { fige Läsionen }\end{array}$ \\
\hline & & & & & & $\begin{array}{l}\text { Nach } 6 \text { Monaten: kein } \\
\text { Rezidiv }\end{array}$ \\
\hline \multirow{2}{*}{$\begin{array}{l}\text { Aktas et al. } \\
(2018),[10]\end{array}$} & \multirow[t]{2}{*}{$65 / w$} & \multirow{2}{*}{$\begin{array}{l}\text { Unterer Rücken } \\
\text { (lumbal) }\end{array}$} & \multirow[t]{2}{*}{ Rückenschmerzen } & \multirow[t]{2}{*}{ Nicht durchgeführt } & Intraläsionale ST & \multirow[t]{2}{*}{ Rückläufige Läsionen } \\
\hline & & & & & Kryotherapie & \\
\hline \multirow{5}{*}{$\begin{array}{l}\text { Tupikowska et al. } \\
\text { (2018), [4] }\end{array}$} & \multirow[t]{5}{*}{$38 / w$} & \multirow[t]{5}{*}{ Regio pubica } & \multirow[t]{5}{*}{ Uterusmyom } & \multirow{5}{*}{$\begin{array}{l}\text { Gemischtzelliges, T-lympho- } \\
\text { zytär dominiertes Infiltrat }\end{array}$} & Orale $\mathrm{AH}$ & \multirow{5}{*}{$\begin{array}{l}\text { Langsam rückläufige } \\
\text { Läsionen }\end{array}$} \\
\hline & & & & & Topische ST & \\
\hline & & & & & Intraläsionale ST & \\
\hline & & & & & $\begin{array}{l}\text { Intramuskuläre } \\
\text { ST }\end{array}$ & \\
\hline & & & & & Kryotherapie & \\
\hline \multirow[t]{2}{*}{$\begin{array}{l}\text { Altamura et al. } \\
(2014),[7]\end{array}$} & \multirow[t]{2}{*}{$50 \mathrm{er} / \mathrm{w}$} & \multirow[t]{2}{*}{ Rücken } & \multirow[t]{2}{*}{ Fibromyalgie } & \multirow[t]{2}{*}{$\begin{array}{l}\text { B-lymphozytär dominiertes } \\
\text { Infiltrat }\end{array}$} & Topische ST & $\begin{array}{l}\text { Nach } 1 \text { Monat: komplett } \\
\text { rückläufige Läsionen }\end{array}$ \\
\hline & & & & & & $\begin{array}{l}\text { Nach } 15 \text { Monaten: kein } \\
\text { Rezidiv }\end{array}$ \\
\hline $\begin{array}{l}\text { Khelifa et al. } \\
\text { (2013), [6] }\end{array}$ & $77 / w$ & $\begin{array}{l}\text { Unterer Rücken } \\
\text { (lumbal) }\end{array}$ & $\begin{array}{l}\text { Rückenschmerzen bei } \\
\text { lumbaler Spinalkanalste- } \\
\text { nose }\end{array}$ & $\begin{array}{l}\text { Gemischt T- und B-lympho- } \\
\text { zytäres Infiltrat }\end{array}$ & $\begin{array}{l}\text { Topische und } \\
\text { intraläsionale ST }\end{array}$ & Rückläufige Läsionen \\
\hline $\begin{array}{l}\text { Choi et al. } \\
\text { (2012), [3] }\end{array}$ & $52 / \mathrm{m}$ & Unterlid bds. & Infraorbitale Augenringe & $\begin{array}{l}\text { Gemischtzelliges, T-lympho- } \\
\text { zytär dominiertes Infiltrat }\end{array}$ & Intraläsionale ST & $\begin{array}{l}\text { Nach } 3 \text { Monaten: rück- } \\
\text { läufige Läsionen }\end{array}$ \\
\hline $\begin{array}{l}\text { Smolle et al. } \\
(2000),[8]\end{array}$ & $56 / w$ & $\begin{array}{l}\text { Unterschenkel } \\
\text { bds. }\end{array}$ & $\begin{array}{l}\text { Chronisch venöse Insuffi- } \\
\text { zienz }\end{array}$ & $\begin{array}{l}\text { B-lymphozytär dominiertes } \\
\text { Infiltrat }\end{array}$ & Intraläsionale ST & $\begin{array}{l}\text { Langsam rückläufige } \\
\text { Läsionen }\end{array}$ \\
\hline
\end{tabular}

Auch für erfahrene Mediziner ist die Diagnose eines Pseudolymphoms oftmals eine Herausforderung und erfordert nicht selten die Korrelation aller Befunde - der Klinik, der Histologie, der Immunhistochemie und der Molekularpathologie. Es ist $\mathrm{zu}$ erwarten, dass mit zunehmender Anwendung der Blutegeltherapie in unterschiedlichen Bereichen der Medizin auch die kutanen Nebenwirkungen häufiger beobachtet werden. Daher sollte bei der differenzialdiagnostischen Abklärung von Pseudolymphomen auch diese seltene Ursache bedacht werden.

\section{Fazit für die Praxis}

- Auch für erfahrene Mediziner ist die Diagnose eines Pseudolymphoms oftmals eine Herausforderung und erfordert nicht selten die Korrelation aller Befunde - der Klinik, der Histologie, der Immunhistochemie und der Molekularpathologie.

- Es ist zu erwarten, dass mit zunehmender Anwendung der Blutegeltherapie in unterschiedlichen Bereichen der Medizin auch die kutanen Nebenwirkungen häufiger beobachtet werden. Daher sollte bei der differenzialdiagnostischen Abklärung von Pseudolymphomen auch diese seltene Ursache bedacht werden.

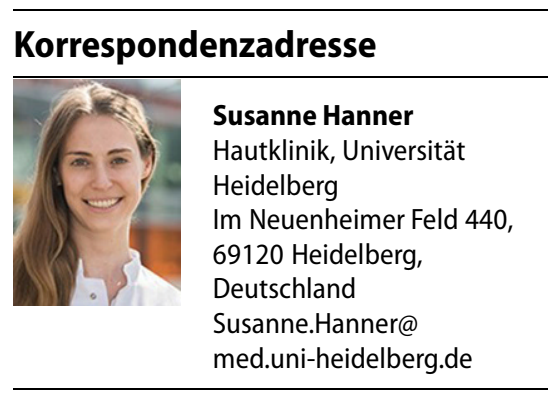

Author Contribution. Alle Autoren hatten vollen Zugang zu den publizierten Daten und tragen die Verantwortung für deren Richtigkeit. Alle Autoren haben bei der Erstellung des Manuskriptes mitgewirkt.

Funding. Open Access funding enabled and organized by Projekt DEAL. 


\section{Einhaltung ethischer Richtlinien}

Interessenkonflikt. S. Hanner, H. Stroh, A. Enk und J. Hoffmann geben an, dass kein Interessenkonflikt besteht.

Für diesen Beitrag wurden von den Autoren keine Studien an Menschen oder Tieren durchgeführt. Für die aufgeführten Studien gelten die jeweils dort angegebenen ethischen Richtlinien. Für Bildmaterial oder anderweitige Angaben innerhalb des Manuskripts, über die Patienten zu identifizieren sind, liegt von ihnen und/oder ihren gesetzlichen Vertretern eine schriftliche Einwilligung vor.

Open Access. Dieser Artikel wird unter der Creative Commons Namensnennung 4.0 International Lizenz veröffentlicht, welche die Nutzung, Vervielfältigung, Bearbeitung, Verbreitung und Wiedergabe in jeglichem Medium und Format erlaubt, sofern Sie den/die ursprünglichen Autor(en) und die Quelle ordnungsgemäß nennen, einen Link zur Creative Commons Lizenz beifügen und angeben, ob Änderungen vorgenommen wurden.

Die in diesem Artikel enthaltenen Bilder und sonstiges Drittmaterial unterliegen ebenfalls der genannten Creative Commons Lizenz, sofern sich aus der Abbildungslegende nichts anderes ergibt. Sofern das betreffende Material nicht unter der genannten Creative Commons Lizenz steht und die betreffende Handlung nicht nach gesetzlichen Vorschriften erlaubt ist, ist für die oben aufgeführten Weiterverwendungen des Materials die Einwilligung des jeweiligen Rechteinhabers einzuholen.

Weitere Details zur Lizenz entnehmen Sie bitte der Lizenzinformation auf http://creativecommons.org/ licenses/by/4.0/deed.de.

\section{Literatur}

1. Sander CA, Flaig MJ (2018) Pseudolymphome. In: Plewig G, Ruzicka T, Kaufmann R, Hertl M (Hrsg) Braun-Falco's Dermatologie, Venerologie und Allergologie. Springer, Berlin, Heidelberg, S 1937-1943

2. Mitteldorf C, Kempf W (2020) Cutaneous pseudolymphoma-A review on the spectrum and a proposal for a new classification. J Cutan Pathol 47(1):76-97

3. Choi Y, Kim S-C (2012) Cutaneous pseudolymphoma induced by Hirudo medicinalis therapy. JDermatol 39(2):195-197

4. Tupikowska M, Woźniak Z, WojciechowskaZdrojowy M, Maj J, Jankowska-Konsur A (2018) Hirudotherapy - a rare cause of pseudolymphoma. Postepy Dermatol Alergol 35(2):225-226

5. Temiz SA, Özer İ, Ataseven A, Dursun R, Fındık S (2019) Cutaneous Pseudolymphoma Due to Hirudotherapy. Turk J Parasitol 43(1):50-52

6. Khelifa E, Kaya G, Laffitte E (2013) Cutaneous pseudolymphomas after leech therapy. J Dermatol 40(8):674-675

7. Altamura D, Calonje E, li Liau J, Rogers M, Verdolini R (2014) Diffuse cutaneous pseudolymphoma due to therapy with medicinal leeches. JAMADermatol 150(7):783-784

8. Smolle J, Cerroni L, Kerl H (2000) Multiple pseudolymphomas caused by Hirudo medicinalis therapy. J Am Acad Dermatol 43(5 Pt 1):867-869
9. Sadati MS, Rezaee M, Ghafarpur S, Aslani FS, Dastgheib L, Jahankhah R (2019) Cutaneous lymphoid hyperplasia induced by Hirudo medicinalis (leeches). J Complement Integr Med 16(4). https:// doi.org/10.1515/jcim-2016-0056

10. Aktas H, Hamidi A, Ertuğrul G, Erol H (2018) A case of cutaneous pseudolymphoma induced by medicinal leech application and review of the literature. Arch Clin Exp Med 3:198-200

11. Sepaskhah M, Yazdanpanah N, Aslani FS, Jahromi MA (2020) Cutaneous pseudolymphoma as a rare adverse effect of medicinal leech therapy: a case report and review of the literature. Cureus 12(4). https://doi.org/10.7759/cureus.7517

12. Sig AK, Guney M, Uskudar Guclu A, Ozmen E (2017) Medicinal leech therapy-an overall perspective. Integr Med Res 6(4):337-343

13. PietrzakA, Kanitakis J, TomasiewiczK, WawrzyckiB, Kozłowska-Łój J, Dybiec E et al (2012) Cutaneous complications of improper leech application. Ann Agric Environ Med 19(4):790-792

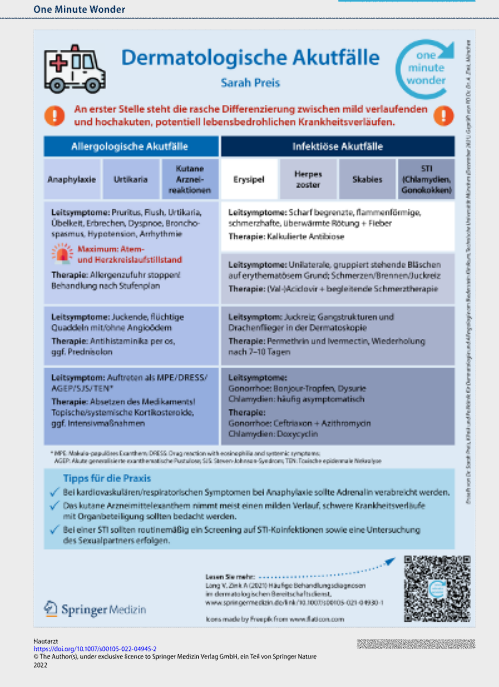

Zum One Minute Wonder dieser Ausgabe

Auch im dermatologischen Bereitschaftsdienst erwartet die Diensthabenden ein großes Spektrum an Erkrankungen. Eine rasche, klinische Differenzierung zwischen mild verlaufenden und hochakuten, potenziell lebensbedrohlichen Krankheitsbildern ist essentiell.

Die Thematik wird in Ausgabe 2/2022 kurz und knapp in einem One Minute Wonder (OMW) aufgegriffen.

OMW zum Beitrag:

Lang V, Zink A (2022) Häufige Behandlungsdiagnosen im dermatologischen Bereitschaftsdienst. Hautarzt 73: $161-170$ www.springermedizin.de/link/ 10.1007/s00105-021-04930-1

Erstellt im Dezember 2021 von:

Dr. Sarah Preis, München

Geprüft und freigegeben im Januar 2022 von: Dr. Sarah Preis und PD Dr. Dr. A. Zink, München

Gestaltung: le-tex Leipzig 